\title{
ORIGINAL
}

\section{CONOCIMIENTO Y USO DE MÉTODOS ANTICONCEPTIVOS POR LA POBLACIÓN FEMENINA DE UNA ZONA DE SALUD}

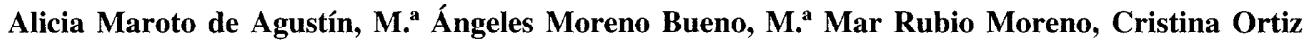 \\ Valle y Francisco Escobar Rabadán.
}

Centro de Salud Zona IV. Albacete.

\section{RESUMEN}

Fundamento: El uso de métodos anticonceptivos está en relación, entre otros, con factores demográficos, sociales, econónicos, educativos e ideológicos. El objetivo de este trabajo es conocer qué métodos anticonceptivos conocen las mujeres en edad fértil, así como la prevalencia de su uso.

Métodos: A partir del listado de tarjeta sanitaria se seleccionaron mediante muestreo sistemático 389 de las $5800 \mathrm{mu}-$ jeres en edad fértil (15-45 años) asignadas a un centro de salud. Previo envío de una carta, comunicando el motivo del estudio, se contactó telefónicamente con ellas para la realización de una encuesta, la cual incluía preguntas acerca del conocimiento y uso de métodos anticonceptivos, características socioculturales y actividad sexual. Las mujeres que no tenían teléfono fueron citadas en el centro de salud.

Resultados: Se contactó con 178 mujeres, de las que participaron 166 (tasa de respuesta de $42.7 \%$ ). De ellas utilizaban algún método anticonceptivo $86(51,8 \%$; IC:44,2$59,4 \%$ ). Sin embargo, entre mujeres con riesgo de embarazo no deseado, la prevalencia de uso era del $70.5 \%$ (IC: $62,4-$ $78,6 \%$ ), destacando el hecho de que en el grupo de mujeres de 40 a 45 años sólo utilizaran anticonceptivos el $45,4 \%$, con una frecuencia significativamente inferior a los otros grupos de edad. Los métodos más conocidos eran el preservativo $(90,4 \%)$, los contraceptivos orales $(89.2 \%)$ y el dispositivo intrauterino $(78,3 \%)$, siendo escaso el conocimiento de otros métodos.

Conclusiones: La tasa de utilización de métodos anticonceptivos en mujeres con riesgo de embarazo no deseado es aceptable. si bien entre 40 y 45 años es llamativamente baja. Los métodos más conocidos son el preservativo, los contraceptivos orales y el dispositivo intrauterino.

Palabras clave: Anticonceptivos orales. Salud de la mujer. Estudio transversal. Encuesta. Dispositivo intrauterino. Salud reproductiva.

Correspondencia:

Francisco Escobar Rabadán

Centro de Salud Zona IV

C/ Seminario, 4

02006-Albacete

Correo electrónico: fescobarr@Albacete.medynet.com

\section{ABSTRACT \\ Knowledge and Use of Contraceptive Methods on the Part of the Female Population in one Health-care District}

Background: The use of contraceptives is related to factors including those of a demographic, social, economic, educational and idcological nature. The purpose of our study is that of ascertaining with what contraceptive methods the women of child-bearing age assigned to a given health care center are familiar, in addition to prevalence of the use thereof.

Methods: Based on the health care card listing, 389 of the 5800 women of child-bearing age ( $15-45$ years old) assigned to the health care center in question were selected by means of a systematic sampling. After sending them a letter notifying them of the reason for this study, they were contacted by telephone to fill out the survey, which included questions regarding their knowledge and use of contraceptive methods, their sociocultural characteristics and sexual activity. Appointments at the health center were made with those women who did not have telephones.

Results: 178 women were contacted, 166 of whom took part in the survey ( $42.7 \%$ response rate). 86 of these women $(51.8 \%$; CI: $44.2-59.4 \%$ ) were using some means of contraception. Nevertheless, among women at risk of unwanted pregnancies, the prevalence of use was $70.5 \%$ (CI: $62.4-78.6 \%$ ), being worthy of special mention the fact that solely $45.4 \%$ of the women within the 40-45 age group were using contraceptives, revealing a frequency significantly lower than the other age groups. The methods known to the greatest degree were the condom $(90.4 \%)$, oral contraceptives $(89.2 \%)$ and the IUD $(78.3 \%)$, the knowledge of other means being scant.

Conclusions: The rate of use of contraceptive methods among women at risk of unwanted pregnancy is acceptable, although strikingly low among the women in the 40-45 age group. The methods known most were the condom, oral contraceptives and the intrauterine device.

Key words: Oral contraceptives. Women's health. Crosssectional study. Survey. Intrauterine device (IUD). Child-bearing health care. 


\section{INTRODUCCIÓN}

La disponibilidad de métodos contraceptivos altamente eficaces ha ofrecido a la mujer la posibilidad de planificar su maternidad, permitiendo un mejor desarrollo personal y una vida sexual más plena. Sin embargo, no todas las mujeres tienen el mismo nivel de conocimiento ni de acceso a ellos.

Se sabe que el uso de métodos anticonceptivos por parte de las mujeres está en relación, entre otros factores, con las conductas sexuales y reproductivas, las percepciones acerca de la contracepción, la edad ${ }^{\mathrm{I}}$, el nivel socioeconómico y educativo ${ }^{2}$ o la paridad $^{3}$. El método seleccionado puede estar en relación además con circunstancias tales como el miedo a las enfermedades de transmisión sexual, el progresivo retraso en la edad de la maternidad, la supresión de recomendaciones médicas en relación con límites de edad para el uso de contraceptivos orales (CO), las creencias de las mujeres en relación con los distintos métodos y sus opiniones sobre los mismos ${ }^{1-4}$ o el nivel de satisfacción en relación con la visita a los profesionales con los que siguen control de dichos métodos ${ }^{5}$.

En los últimos años se han publicado en nuestro país diferentes estudios sobre el uso de métodos anticonceptivos, predominando los que seleccionan poblaciones clínicas, bien sea en centros de orientación familiar ${ }^{6,7}$ o en consultas de atención primaria ${ }^{8.9}$, con el consiguiente sesgo que entraña la selección de mujeres que están siendo atendidas en servicios sanitarios. Por otra parte, los escasos estudios de base poblacional se centran en el uso de un método en particular ${ }^{10}$ $o$ en determinados grupos de edad ${ }^{1}$. Finalmente, no disponemos de datos relativos a nuestro medio, si exceptuamos alguna aproximación parcial ${ }^{12}$.

Estas razones nos han llevado a realizar el presente estudio, en el que nos hemos planteado como objetivo conocer la prevalencia de uso y los tipos de métodos anti- conceptivos utilizados por las mujeres de nuestra zona de salud, así como las características socioculturales que se asocian con este uso. Por otra parte, queremos conocer qué saben estas mujeres acerca de los mismos.

\section{SUJETOS Y MÉTODOS}

Para dar respuesta a estas cuestiones, diseñamos un estudio observacional de tipo descriptivo transversal, en el que la población diana eran las mujeres en edad fértil (15-45 años) asignadas al centro de salud Zona IV de Albacete. A partir de los listados de la tarjeta sanitaria de los 10 cupos médicos del centro, se identificaron 5.800 mujeres entre estas edades, de las que se seleccionó una muestra de 389 por medio de un muestreo sistemático, con arranque aleatorio. El tamaño muestral se calculó para una frecuencia esperada del $50 \%$, una precisión de $\pm 5 \%$ y un nivel de confianza del $95 \%$.

Previo envío de una carta, comunicando a las mujeres seleccionadas el motivo de nuestro estudio, se contactó con ellas telefónicamente a lo largo del mes de julio de 1997, para la realización de una encuesta que incluía preguntas abiertas subre el conocimiento acerca de métodos anticonceptivos y el uso de los mismos: utilización o no de estos, y, en caso de respuesta afirmativa, cuál; tipo de controles, frecuencia y dónde eran realizados; y posibles complicaciones. El cuestionario incluía asimismo preguntas referidas a características socioculturales de la mujer (edad, estado civil, nivel de estudios, ocupación, situación laboral) y, en su caso, de su pareja o de la persona que aportara los ingresos principales de la familia; sobre su actividad sexual (si tenía relaciones $\mathrm{y}$, en su caso, edad de inicio y frecuencia de las mismas). Siguiendo la clasificación propuesta por Domingo y Marcos ${ }^{13}$, se estableció el nivel económico de las mujeres, distinguiendo entre clase social media-alta (si ella o el marido o cabeza de familia, en su caso, pertenecían a las clases I -directivos, 
altos funcionarios, profesionales liberales, técnicos superiores - o II - directivos y propietarios gerentes de comercio y de los servicios personales, otros técnicos no superiores y artistas y deportistas-) y mediabaja y baja (para las demás ocupaciones). Se clasificó a las mujeres en «expuestas» y «no expuestas» a partir del concepto introducido por Riphagen y Lehert ${ }^{14}$, según el cual se considerarían como «mujeres expuestas» aquellas que no han tenido problemas de fertilidad, sexualmente activas, no embarazadas y que no quieran estarlo en el período del estudio.

Se acordó realizar un máximo de tres intentos, en horas diferentes, para contactar con cada mujer; si tras estos tres intentos no se obtenía respuesta se consideraba no localizada.

No tenían teléfono 79 de las mujeres de nuestra muestra, por lo que fueron citadas en las consultas del centro de salud para la realización de la encuesta.

El análisis de los datos fue realizado por medio del paquete estadístico EPIINFO, e incluyó comparación de variables cualitativas por medio de la prueba de $\mathrm{ji}^{2}$ y de variables cuantitativas por la $t$ de Student.

\section{RESULTADOS}

Se consiguió contactar con 178 mujeres, de las que contestaron 166 (tasa de respuesta de $42,7 \%$ ), las cuales tenían una media de edad de 29,7 años (DE: 8,1), con un rango entre 15 y 45 años. Se localizaron a través del teléfono 171 mujeres, de las que 12 no quisieron responder. De las 79 citadas en el centro de salud acudieron 7 .

Utilizaban algún método anticonceptivo 86 mujeres $(51,8 \%$; IC: $44,2-59,4 \%)$, mientras que 73 no los usaban, 2 estaban buscando embarazo, 3 estaban embarazadas o en el postparto y 2 no contestaron a esta pregunta. Una mujer que dijo usar como méto- do anticonceptivo el coitus interruptus fue incluida en el grupo de las no utilizadoras.

Al analizar las «mujeres expuestas» encontramos que utilizaban anticonceptivos el 70,5\% (IC: $62,4-78,6 \%$ ). Como podemos ver en la tabla 1, de las 20 adolescentes de nuestro estudio sólo dos mantenían relaciones sexuales, de las cuales una usaba anticoncep-

Tabla 1

Características sociodemográficas de las mujeres y uso de métodos anticonceptivos

\begin{tabular}{|c|c|c|c|c|}
\hline & $\begin{array}{l}\text { Uti- } \\
\text { liza }\end{array}$ & $\begin{array}{l}\text { No } \\
\text { uti- } \\
\text { liza }\end{array}$ & $\begin{array}{l}\text { No ex- } \\
\text { puestas }\end{array}$ & Total \\
\hline \multicolumn{5}{|l|}{ Edad * } \\
\hline 15-19 años & 1 & 1 & 18 & 20 \\
\hline 20-29 años & 30 & 11 & 21 & 62 \\
\hline 30-39 años & 45 & 12 & 3 & 60 \\
\hline $40-45$ años & 10 & 12 & & 22 \\
\hline \multicolumn{5}{|l|}{ Estado Civil } \\
\hline Soltera & 19 & 5 & 38 & 62 \\
\hline Casada & 67 & 27 & 4 & 98 \\
\hline Separada & & 4 & & 4 \\
\hline \multicolumn{5}{|l|}{ Estudios } \\
\hline Sin estudios & 7 & 3 & & 10 \\
\hline Primar. Incompletos & 4 & 2 & & 6 \\
\hline Primarios & 44 & 23 & 17 & 84 \\
\hline Secundarios & 22 & 6 & 17 & 45 \\
\hline Titulados med.-sup. & 9 & 2 & 8 & 19 \\
\hline \multicolumn{5}{|l|}{ Ocupación } \\
\hline \multicolumn{5}{|l|}{ Trabajo rcmuncrado } \\
\hline Directivas & 3 & 1 & 1 & 5 \\
\hline Técnicas & 8 & 3 & 2 & 13 \\
\hline Cuadros intermedios & 27 & 8 & 12 & 47 \\
\hline Trabaj. Manuales & 3 & 2 & 2 & 7 \\
\hline Estudiantes & 2 & 1 & 20 & 23 \\
\hline Amas de casa & 40 & 21 & 3 & 64 \\
\hline No clasificables & 2 & & 1 & 3 \\
\hline No consta & 1 & & 1 & 2 \\
\hline \multicolumn{5}{|l|}{ Nivel económico } \\
\hline Medio-alto y alto & 22 & 6 & 11 & 39 \\
\hline Medio-bajo y bajo & 64 & 30 & 26 & 120 \\
\hline No consta & & & 5 & 5 \\
\hline Total & 86 & 36 & 42 & 164 \\
\hline
\end{tabular}

$* \mathrm{ji}^{2}=8,6 ; \mathrm{p}<0,05$.

Resto no significativo (NS). 
tivos; especialmente llamativo resulta que en el grupo de mujeres de 40 a 45 años sólo utilizaran anticonceptivos el $45,4 \%$, en una frecuencia significativamente inferior a los otros grupos de edad. No se encontraron diferencias significativas en cuanto a la utilización o no de anticonceptivos según el estado civil, el nivel de estudios, la ocupación o el nivel económico. Las mujeres que usaban anticonceptivos tenían una media de relaciones sexuales por mes de 8,4 (DE: $5,6)$, que no difería significativamente de las 8,2 (DE: 7,7) de las que no los usaban. Tampoco existían diferencias en la media de edad de inicio de estas relaciones: 19,9 (DE: 4,3) frente a 20,5 años (DE: 2,7).

El método más usado era el preservativo, seguido de los métodos quirúrgicos y los $\mathrm{CO}$, como queda reflejado en la tabla 2 , en la que se recogen los datos correspondientes a las características sociodemográficas de las mujeres en función del tipo de método anticonceptivo que utilizaban. Los CO tenían una utilización significativamente más frecuente en mujeres menores de 30 años y menor en las de esta edad o mayores; por contra, en este grupo de edad era significativamente más frecuente el uso de un método quirúrgico, frente a las más jóvenes. Los métodos de barrera eran significativamente más usados por las mujeres solteras y menos por las casadas, tendencia opuesta a la encontrada para el resto de los métodos considerados globalmente. No se encontraron diferencias en relación con los estudios de la mujer o su nivel económico.

Seguían algún control en relación con el uso de anticonceptivos 39 mujeres, mientras que no lo hacían $43 ; 4$ no contestaron a esta pregunta. Todas las mujeres que usaban $\mathrm{CO}$ o dispositivo intrauterino (DIU) seguían controles periódicos, consistentes en la práctica de citología cervical en las usuarias de DIU y en el $86,7 \%$ de las de CO; de éstas el $66,7 \%$ se hacían también analítica. El seguimiento se realizaba preferentemente en el Centro de Orientación Familiar $(50 \%$ de las usuarias de $\mathrm{CO}$ y $75 \%$ de las de DIU), quedando en segundo lugar las consultas
Tabla 2

Características sociodemográficas de las mujeres y tipo de método anticonceptivo utilizado

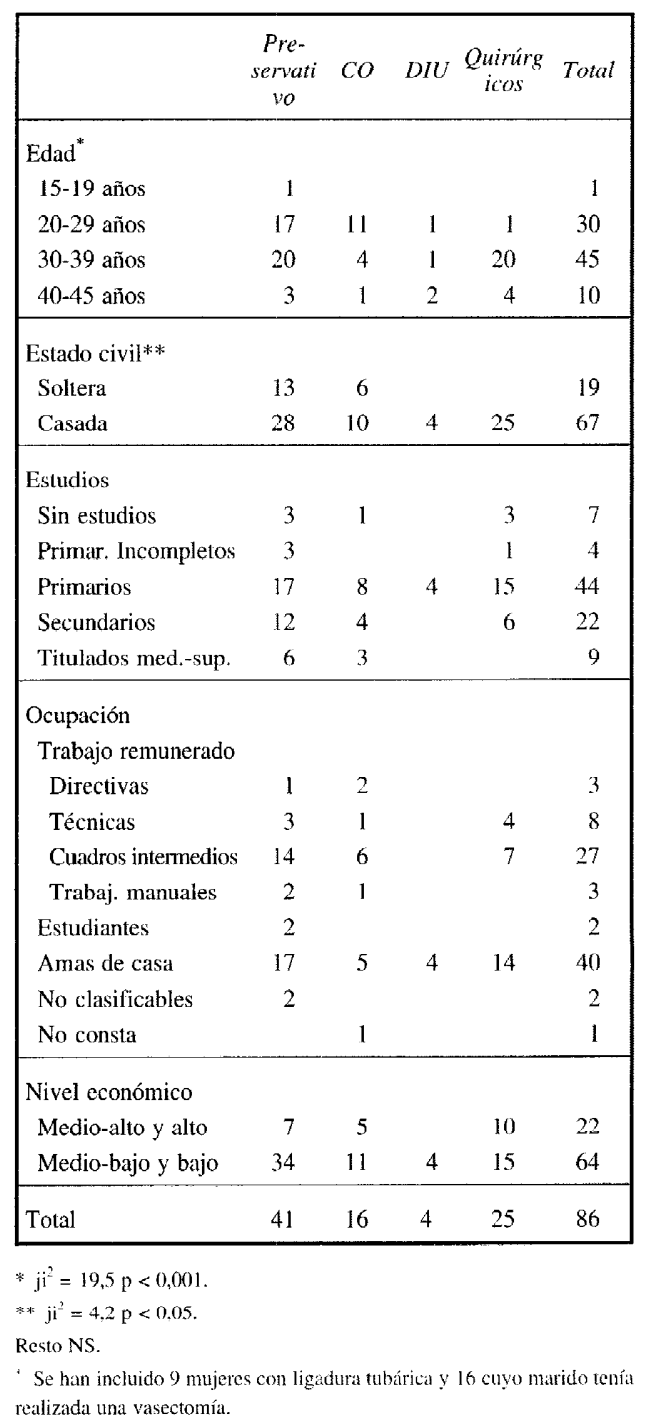

privadas de ginecología $(31,2 \%$ de las que usaban $\mathrm{CO}$ y $25 \%$ de las de DIU). En el centro de salud se controlaban el $12,5 \%$ de las usuarias de CO. Para todas las usuarias de DIU, así como para el 78,6\% de las de CO había pasado menos de un año desde el último control. No se encontraron diferencias significativas en cuanto al lugar donde se controlaban, el tipo de controles o la fre- 
cuencia de los mismos en función del nivel económico de las mujeres.

Las mujeres de nuestro estudio que utilizaban anticonceptivos referían escasas complicaciones: una usuaria de preservativo refería como tal la rotura del mismo; dos de las que utilizaban $\mathrm{CO}$ refirieron cefalea y una además aumento de peso y dismenorrea: una usuaria de DIU refería hipermenorrea; y una mujer con ligadura tubárica se quejaba de disminución de la libido.

Las mujeres que tomaban $\mathrm{CO}$ tenían una media de relaciones sexuales al mes de 11,0 (DE: 4,9), significativamente superior a las 6,2 (DE: 3,8) de las usuarias de preservativo $(t=7,3, \mathrm{p}<0,05)$. No existían difcrencias significativas en la frecuencia de relaciones sexuales con respecto a las usuarias de otros métodos. Tampoco se encontraron diferencias significativas en la edad de inicio de las relaciones sexuales entre las usuarias de los distintos métodos.

En la figura 1 podemos ver cuántas mujeres decían conocer los diferentes métodos anticonceptivos. Los métodos más conocidos eran el preservativo $(90,4 \%)$, los $\mathrm{CO}$ $(89,2 \%)$ y el DIU $(78,3 \%)$. Como se evidencia en las figuras 2 y 3 , las mujeres más jóvenes y las que no convivían con pareja conocían mejor los métodos de barrera, tanto

Figura 1

Conocimiento sobre métodos anticonceptivos expresado por las mujeres de nuestro estudio

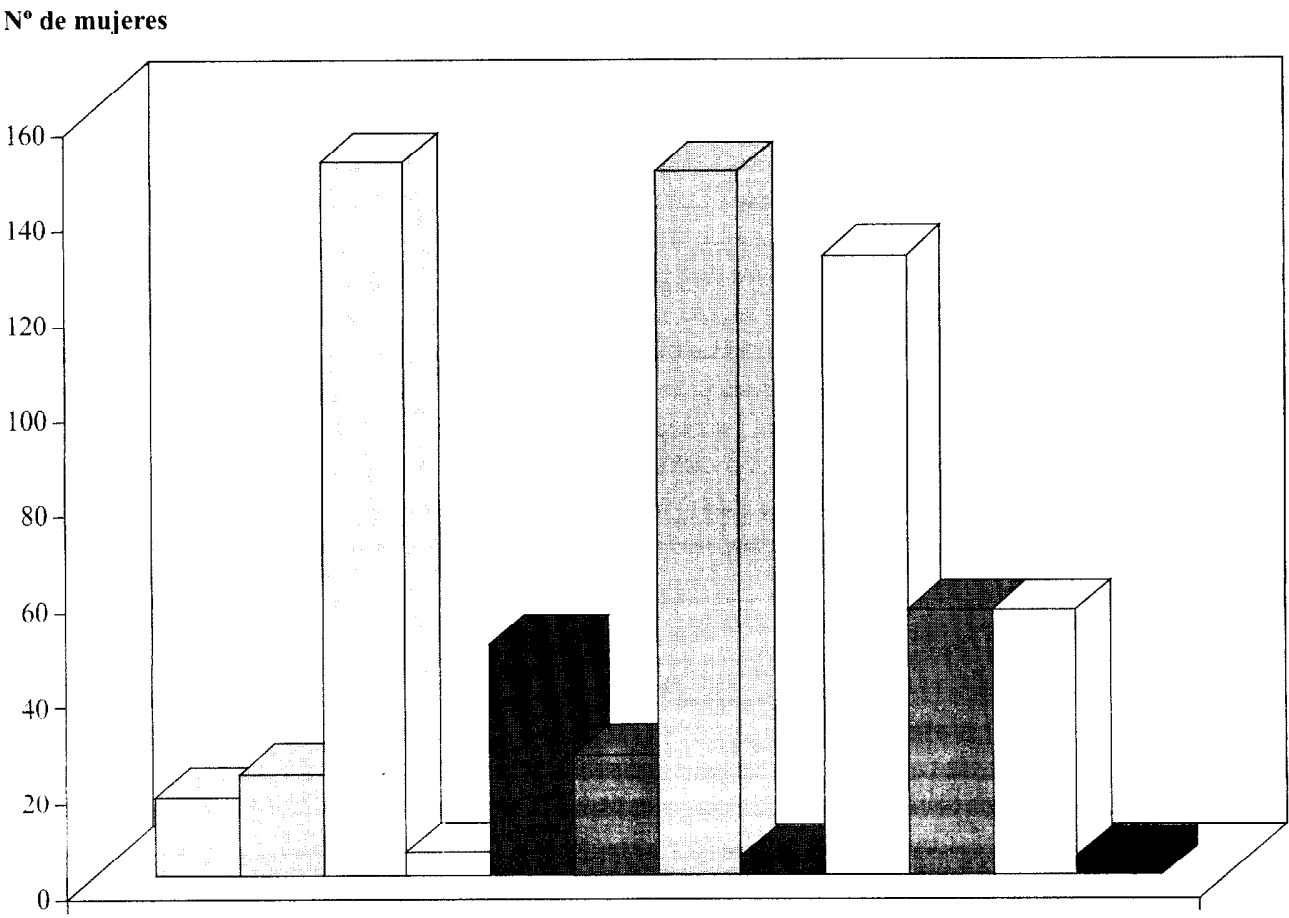

\begin{tabular}{|lll|}
\hline$\square$ Coitus interruptus & $\square$ Métodos naturales & $\square$ Preservativo \\
$\square$ Preservativo femenino & $\square$ Diafragma & Espermicidas \\
$\square$ Contraceptivos orales & $\square$ Contraceptivos inyectables & $\square$ Dispositivo intrauterino \\
Ligadura tubárica & $\square$ Vasectomia & $\mathbf{\square}$ Abstinencia \\
\hline
\end{tabular}


Figura 2

Conocimiento de métodos anticonceptivos en relación con la edad

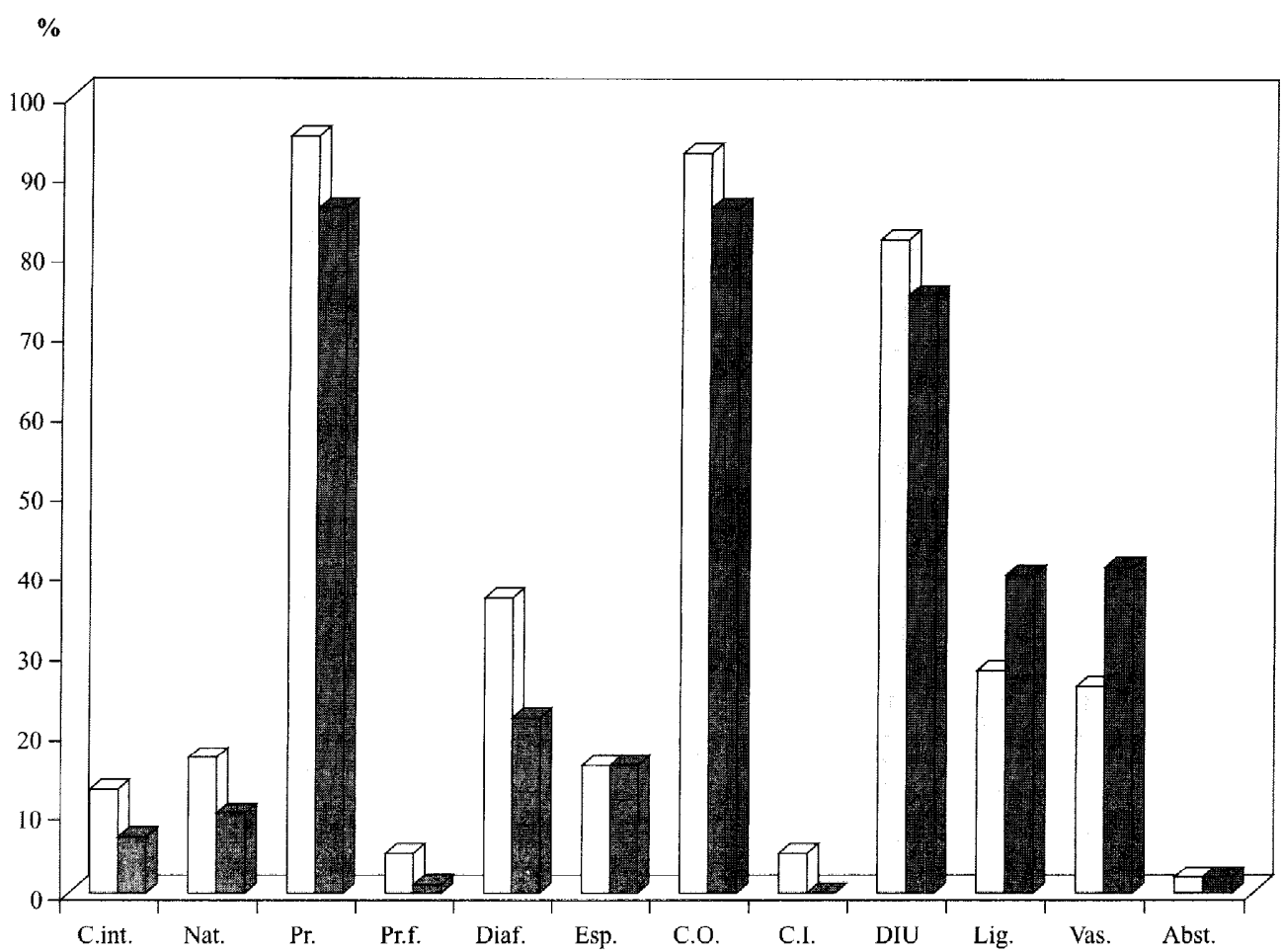

$>0=30$ años

Nota.- C.int: coitus interruptus (NS) Nat: : Métodos naturales (NS) Pr.: Preservativo $\left(\mathrm{ji}^{2}=4,4 ; \mathrm{p}<0,05\right)$ Pr.f.: Preservativo femenino (NS)
Diaf.: Diafragma $\left(\mathrm{ji}^{2}=4.9 ; \mathrm{p}<0,05\right)$ Esp.: Espermicidas (NS)

C.O.: Contraceptivos orales (NS)

C.I.: Contraceptivos inyectables (NS)
DIU: Dispositivo intrauterino (NS) Lig.: Ligadura tubárica (NS) Vas.: Vasectomía $\left(\mathrm{ji}^{2}=3,9 ; \mathrm{p}<0,05\right)$ Abst.: Abstinencia (NS) el preservativo como el diafragma, mientras que la vasectomía era mejor conocida por las de mayor edad, especialmente en el grupo de 30-39 años, en el que un 48,3\% hablaban de ella, frente al $30,25,4$ y $21,7 \%$ para los grupos de 15-19, 20-29 y 40-45 años $\left(\mathrm{ji}^{2}: 9,3, \mathrm{p}<0,05\right)$. En general las mujeres con estudios secundarios o superiores tenían un mejor conocimiento de los distintos métodos anticonceptivos (figura 4). No se encontraron diferencias en el conocimiento de métodos anticonceptivos según la ocupación o el nivel económico de las mujeres, así como tampoco en relación con la edad de inicio de las relaciones sexuales.

\section{DISCUSIÓN}

Un $51,8 \%$ de las mujeres en edad fértil que participaron en nuestro estudio utilizaban algún método anticonceptivo, alcanzando el $70,5 \%$ cuando consideramos «mujeres expuestas». El método más usado era el pre- 
Figura 3
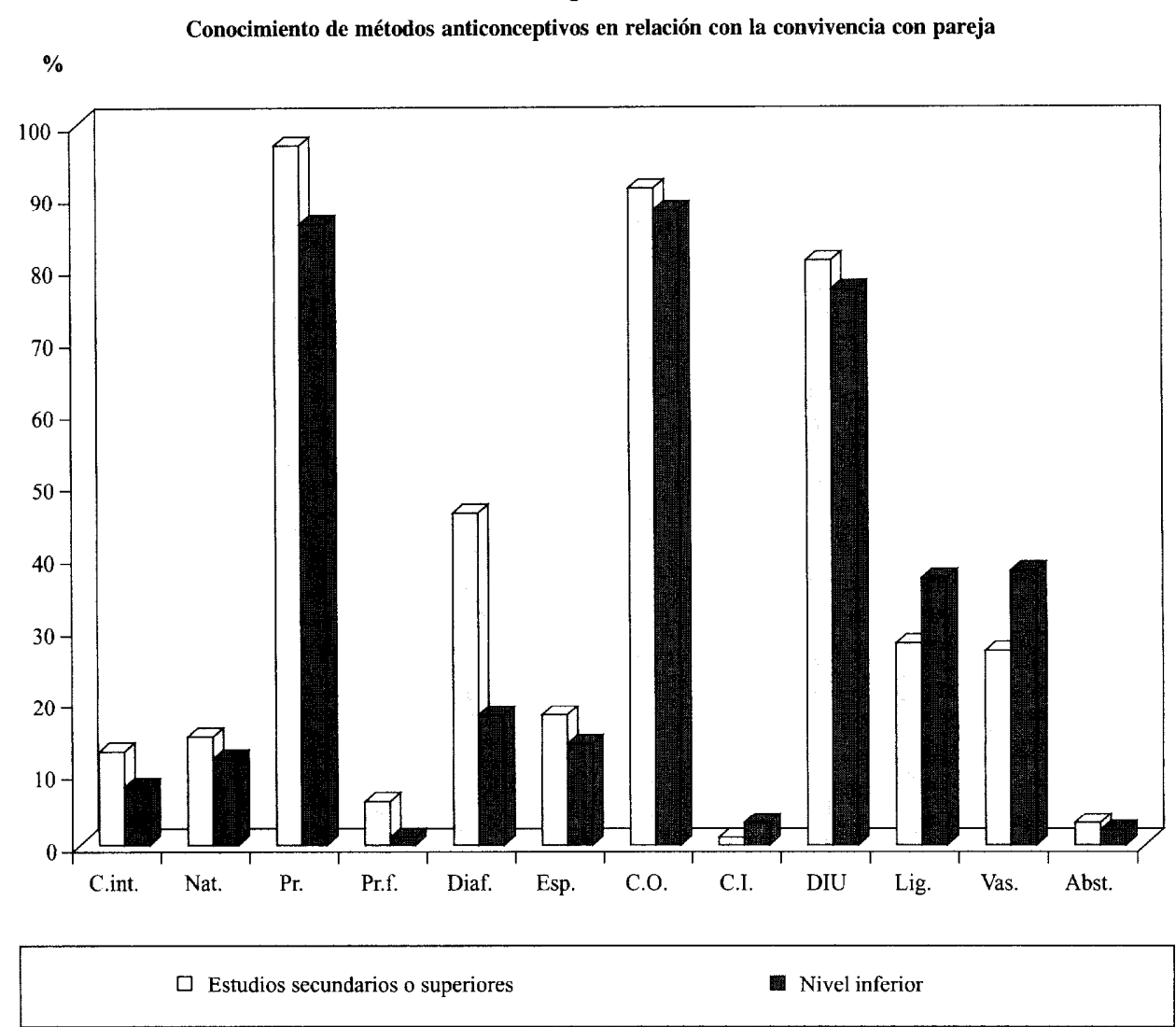

Nota.- C.int.: coitus interruptus (NS) Nat:: Métodos naturales (NS) Pr.: Preservativo $\left(\mathrm{ji}^{2}=5,7 ; \mathrm{p}<0,05\right)$ Pr.f.: Preservativo femenino (NS)
Diaf.: Diafragma $\left(\mathrm{ji}^{2}=15,1 ; \mathrm{p}<0,001\right)$

Esp.: Espermicidas (NS)

C.O.: Contraceptivos orales (NS)

C.I.: Contraceptivos inyectables (NS)
DIU: Dispositivo intrauterino (NS)

Lig.: Ligadura tubárica (NS)

Vas.: Vasectomía (NS)

Abst:: Abstinencia (NS) servativo, seguido de los métodos quirúrgicos y los CO.

Nuestros datos están en concordancia con los recogidos por Serrano y cols. ${ }^{12}$ en su estudio acerca del uso de métodos anticonceptivos por mujeres lactantes en nuestra ciudad, en el que un $69 \%$ de ellas refería utilizar anticonceptivos previamente a su última gestación, con un predominio del uso de los métodos de barrera. Debemos ser prudentes, sin embargo, al establecer estos paralelismos, ya que se trata de un estudio diseñado con otro objetivo y que aborda un grupo tan específico como es el de las mujeres en período de lactancia, circunstancia que además determina que la muestra tenga unas características sociodemográficas muy distintas a la de nuestro estudio.

Como señalábamos en la introducción, la mayoría de los estudios realizados en nuestro país sobre uso de anticonceptivos seleccionan a las mujeres participantes en centros sanitarios, ya sean consultas de atención primaria o de planificación familiar. Los resultados obtenidos en estos estudios muestran notables diferencias con los nuestros. Así, Sánchez Pérez y cols. ${ }^{8}$, al estudiar a un grupo de 371 mujeres de 15 a 45 años que acudían a una consulta de atención primaria y que mantenían relaciones sexuales, encontraron 
Figura 4

Conocimiento de métodos anticonceptivos en relación con el nivel de estudios

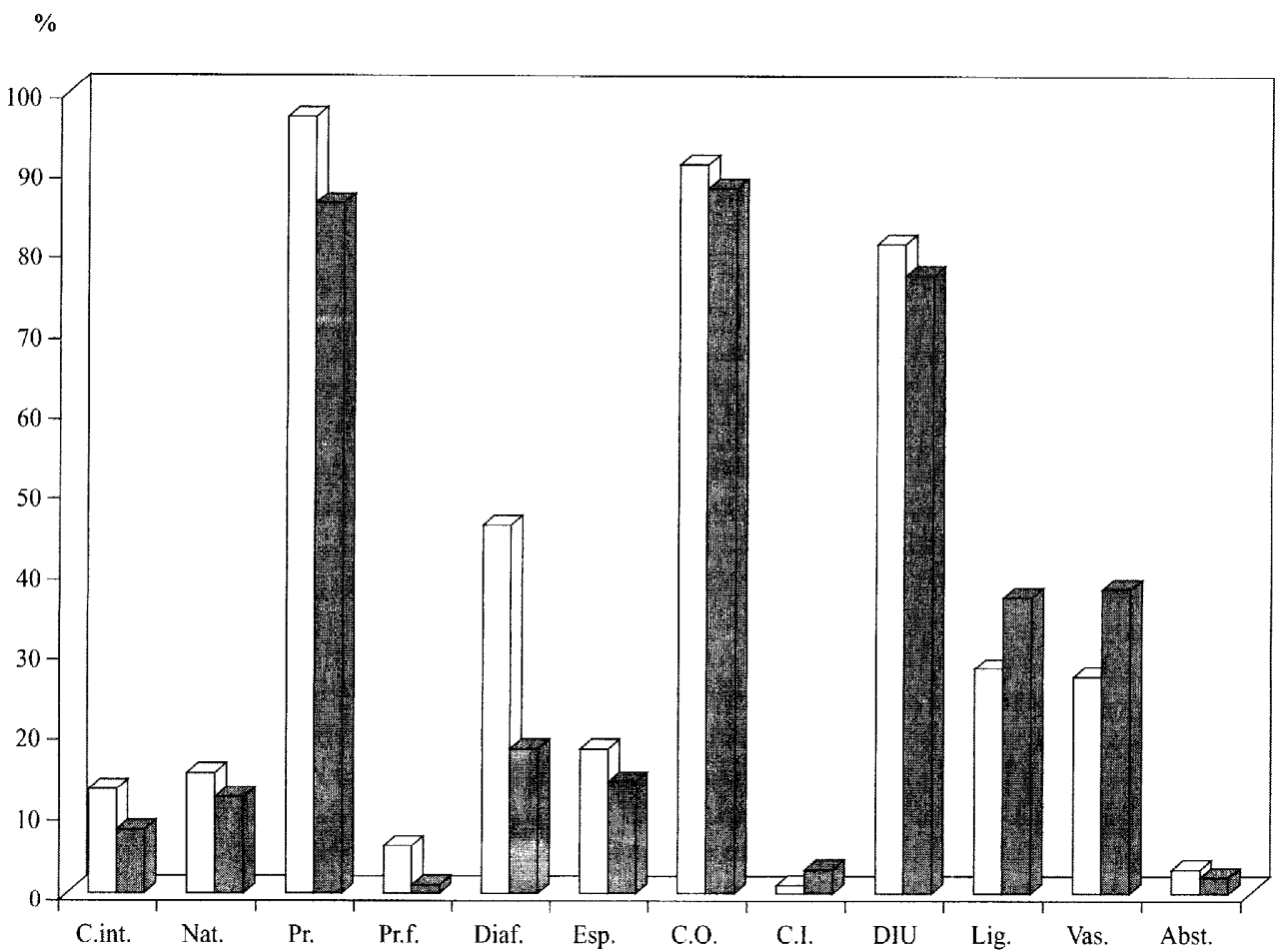

Nota.- C.int:: coitus interruptus $\left(\mathrm{ji}^{2}=11,1 ; \mathrm{p}<0,00 \mathrm{H}\right)$

Nat.: Métodos naturales $\left(\mathrm{ji}^{2}=15,5 ; \mathrm{p}<0,001\right)$

Pr.: Preservativo $\left(\mathrm{ji}^{2}=5,3 ; \mathrm{p}<0,05\right)$

Pr.f.: Preservativo femenino (NS)

Diaf.: Diafragma $\left(\mathrm{ji}^{2}=30,4 ; \mathrm{p}<0,001\right)$

Esp.: Espennicidas $\left(\mathrm{ji}^{2}=14,9: \mathrm{p}<0,001\right)$

que sólo 41 no usaban ningún método, si bien 31 utilizaban el coitus interruptus; el método más utilizado era el preservativo, seguido de $\mathrm{CO}$ y los métodos quirúrgicos, con una distribución similar a la de nuestro estudio. Esta distribución en el uso de los distintos métodos es, sin embargo, notablemente distinta a la encontrada por Rodríguez González y cols. ${ }^{9}$ : el $20,3 \%$ de un grupo de 176 mujeres de 14-52 años que acudían a una consulta de atención primaria
C.0.: Contraceptivos orates (NS)

C.I.: Contraceptivos inyectables (NS)

DIU: Dispositivo intrauterino $\left(\mathrm{ji}^{2}=7,5 ; \mathrm{p}<0,01\right)$

Lig.: Ligadura tubárica (NS)

Vas.: Vasectomía $\left(\mathrm{j}^{2}=7,4 ; \mathrm{p}<0,05\right)$

Abst.: Abstinencia (NS)

usaban $\mathrm{CO}$, mientras que el $18,3 \%$ usaban como método anticonceptivo el coito interrumpido, quedando el preservativo en tercer lugar con un $17 \%$. Por otra parte, en estudios realizados en consultas de planificación familiar, como el llevado a cabo por Adán Gil y cols. ${ }^{6}$ en mujeres mayores de 19 años que acudían solicitando un método anticonceptivo a un centro de orientación familiar, el $56,67 \%$ usaban anticoncepción hormonal, seguida por el DIU $(42,94 \%)$. 
Como señalábamos en la introducción, estos estudios presentan un sesgo de selección que limita su generalización, y que es especialmente llamativo en el caso de las mujeres que acuden a una consulta de planificación familiar, ya que de forma habitual lo hacen para seguir control médico por alguno de los métodos anticonceptivos que así lo requieren.

En nuestro estudio hemos seleccionado una muestra de la población de mujeres en edad fértil asignadas a nuestro centro de salud, y que podríamos considerar como asimilable a población general si tenemos en cuenta la cobertura casi universal del Sistema Nacional de Salud y que su adscripción es independiente de que hayan o no consultado alguna vez.

Una posible limitación de nuestro trabajo se encuentra en la tasa de respuesta, al haber participado en el mismo sólo el $42,7 \%$ de las mujeres seleccionadas. Sin embargo, lo consideramos bastante aceptable para el tipo de encuesta realizada, en donde la falta de respuesta está relacionada sobre todo con la imposibilidad de contactar telefónicamente con la mujer. En cambio, sí que puede suponer un sesgo la falta de asistencia a la cita concertada en el centro de salud de las mujeres que no tenían teléfono, ya que podrían corresponder a un grupo económicamente menos favorecido.

Otra diferencia en relación con algunos de los estudios referidos anteriormente, ha sido el no considerar en nuestro análisis el coitus interruptus como método anticonceptivo, por tratarse de un método muy poco seguro y para evitar el posible sesgo de que algunas mujeres que lo estuvieran utilizando no lo consideraran como tal, lo que se vería favorecido por el hecho de plantear preguntas abiertas. Seleccionamos este tipo de preguntas para no inducir la respuesta de las mujeres, asumiendo el posible inconveniente de que se perdiera información, lo que en el caso de métodos anticonceptivos de contrastada eficacia y de in- dudable indicación médica sería menos probable.

En nuestro análisis tiene escaso relieve el grupo de adolescentes, ya que sólo 2 de ellas mantenían relaciones sexuales, mientras que es muy llamativo el bajo porcentaje de uso de anticonceptivos entre las mujeres de 40 a 45 años. Este dato tiene una extraordinaria relevancia por la posibilidad que entraña de embarazos no deseados en este grupo de edad de especial riesgo.

En cuanto al método elegido según la edad, el estado civil o la frecuencia de relaciones sexuales, nuestros resultados están en la línea de lo esperado atendiendo a las características de los distintos métodos, como sería un mayor uso de los quirúrgicos conforme aumenta la edad de las mujeres o la preferencia por el preservativo en mujeres solteras o con baja frecuencia de relaciones sexuales. Así encontramos que el uso de los $\mathrm{CO}$ predominaba en las mujeres menores de 30 años y el de los métodos quirúrgicos en las mayores de esta edad, siendo el uso del preservativo más frecuente en mujeres solteras que en casadas. Por otra parte, las mujeres que tomaban $\mathrm{CO}$ tenían una media de relaciones sexuales al mes significativamente superior a las usuarias de preservativo.

Existen diferencias muy llamativas al comparar nuestros datos con los de otros países occidentales, tanto en la frecuencia de uso de anticonceptivos como en los tipos de métodos más utilizados. Así en Alemania sólo un $3 \%$ de mujeres expuestas no usan ningún método anticonceptivo, siendo predominante la utilización de $\mathrm{CO}^{4}$, al igual que ocurre en Francia, donde los utilizan un $40 \%$ de las mujeres de 20 a 44 años ${ }^{15}$, o en el Reino Unido, quedando en estos países el uso del preservativo en segundo lugar ${ }^{16}$. La preferencia por los $\mathrm{CO}$ se da también en otros países europeos, como Italia ${ }^{17}$ o Suecia $^{18}$, donde son utilizados, respectivamente, por un 30 y un $33 \%$ de las «mujeres expuestas». En EEUU, con una frecuencia de uso de anticonceptivos en «mujeres expuestas» 
de alrededor del $90 \%$, los $\mathrm{CO}$ han sido el método preferido por las mujeres menores de 30 años, aunque globalmente existe un predominio de los métodos quirúrgicos ${ }^{\mathrm{l}}$; sin embargo, entre 1988 y 1995 se ha observado una disminución de usuarias de $\mathrm{CO}$, que han pasado del 31 al $27 \%$, y un aumento en las de preservativo, del 15 al $20 \%$, especialmente en mujeres solteras y en menores de 25 años ${ }^{19}$. Esta preferencia por $\mathrm{CO}$ en mujeres jóvenes, con predominio en conjunto de los métodos quirúrgicos la encontramos asimismo en Australia ${ }^{20}$.

La baja frecuencia de uso de CO en nuestra zona de salud está en consonancia con las tendencias de uso de los mismos en nuestro país, como se puso de manifiesto en la Encuesta Nacional de Salud de España de $1993^{10}$, en la que sólo un $4,6 \%$ de las mujeres referían haber consumido $\mathrm{CO}$ en las dos semanas previas a la realización de la cncuesta.

El método anticonceptivo más conocido entre las mujeres que participaron en nuestro estudio fue el preservativo, seguido por los CO y el DIU. Llama la atención el escaso conocimiento de otros métodos, especialmente los quirúrgicos, a pesar de ser muy utilizados. Pensamos que en parte este bajo conocimiento de los métodos puede estar relacionado, como ya comentamos anteriormente, con la realización de preguntas abiertas, mientras que para alguno de ellos, como los naturales o el coitus interruptus, se puede deber a que no se les haya tenido en cuenta por considerarlos poco eficaces. Sin embargo, estos factores no parecen haber sido tan determinantes en otros estudios realizados en nuestro país ${ }^{8,9}$, en los que en general las mujeres muestran un mejor conocimiento de los diferentes métodos, si bien hemos de señalar que están realizados sobre pacientes que asisten a consultas de atención primaria, y por tanto puede existir una sobrerrepresentación de mujeres con una mayor motivación por los temas relacionados con la salud. Destaca en estos estudios, al igual que en el nuestro, el mayor conocimiento de preservativo, $\mathrm{CO}$ y DIU, métodos anticonceptivos que podríamos considerar que son los que gozan de mayor popularidad entre las mujeres de nuestro país.

En cualquier caso, pensamos que existe una necesidad de formación en estos temas en las mujeres de nuestra zona de salud. De hecho, las mujeres con nivel educativo superior han demostrado en nuestro estudio un mejor conocimiento de la mayoría de los métodos. Llama igualmentc la atención el mayor conocimiento de los métodos barrera entre las mujeres más jóvenes, lo que podríamos atribuir a las campañas de prevención de las enfermedades de transmisión sexual, especialmente el sida.

Para concluir queremos resaltar que, aunque la tasa de utilización de métodos anticonceptivos en «mujeres expuestas» es aceptable, en el grupo de edad de 40 a 45 años es llamativamente baja. Por otra parte, aunque el preservativo, los CO y el DIU son bien conocidos, es limitado el conocimiento acerca de otros métodos por parte de las mujeres de nuestra zona de salud.

\section{BIBLIOGRAFÍA}

1. Trussell J, Vaughan B. Contraceptive use projections: 1990 to 2010. Am J Obstet Gynecol 1992; 167: $1160-1164$.

2. Tyrer LB. Obstacles to use of hormonal contraception. Am J Obstet Gynecol 1994; 170: 1495-1498.

3. Nafo F, Wollast E, Serniclaes W, Vekemans M. Ignorances et préjugés dans l'utilisation de méthodes contraceptives. Rev Med Brux 1993; 14: 216220 .

4. Oddens BJ, Visser AP, Vemer HM, Everaerd WTAM. Contraceptive use and attitudes in reunified Germany. Eur J Obstet Gynecol Reprod Biol 1994; 57: 201-208.

5. Forrest JD, Frost IJ. The family planning attitudes and experiences of low-income women. Fam Plann Perspect 1996; 28: 246-255.

6. Adán Gil FM, Guillén Antón MV, Moral Serrano $S$, Fondevila Frutos MJ. Perfil de usuaria y tolerancia de los principales mélodos anticonceptivos 
en la atención primaria: anticonceptivos hormonales $(\mathrm{ACH})$, dispositivo intrauterino (DIU) y diafragma. Aten Primaria 1995; 15: 101-104.

7. Ruiz Jiménez MA, Martínez Nieto JM, Fernández García JR, Pavón Lebrero R, Cano Fernández MC. Anticoncepción en adolescentes incluidas en un programa de planificación familiar. Aten Primaria 1997; 19: 455-458.

8. Sánchez Pérez MR, Gálvez Ibáñez M, Martín Robles M, García Mirón C, García Fernández E, Jiménez Liñán RM. Influencia del nivel socioeconómico y cultural en el uso de métodos anticonceptivos. Aten Primaria 1994: 13: 426-430.

9. Rodríguez González A, García Soldevila M, Santiveri Villuendas A, Saus Arús M, Cortés Torres J, López Amorós M. Planificación familiar: una necesidad en atención primaria. Aten Primaria 1991; 8: 387-391.

10. Prada C, del Río MC, Alvarez FJ. Consumo de anticonceptivos y hábito tabáquico en las mujeres españolas. Aten Primaria 1996; 18: 442-445.

11. Parera N, Suris JC. Sexuality and contraception in adolescents from Barcclona, Spain. J Pediatr Adolesc Gynecol 1997; 10: 153-157.

12. Serrano A, Lacal JF, Jiménez F, Lorenzo I, López-Torres J. Utilización de métodos anticonceptivos durante el período de lactancia. Aten Primaria $1996 ; 18: 368-372$.
13. Domingo A, Marcos J. Propuesta de un indicador de la clase social basado en la ocupación. Gac Sanit 1989; 3: 320-326.

14. Riphagen FE, Lehert P. A survey of contraception in five West European countries. J Biosoc Sci 1989; 21: 23-46.

15. Toulemon L, Leridon H. Contraceptive practices and trends in France. Fam Plann Perspect 1998; 30: 114-120.

16. Fuchs N, Prinz H, Koch U. Attitudes to current oral contraceptive use and future developments: the women's perspective. Eur J Contracept Reprod Health Care 1996; 1: 275-284.

17. Oddens BJ. Contraceptive use and attitudes in Italy 1993. Human Reproduction 1996; 11: 533-539.

18. Oddens BJ, Milsom I. Contraceptive practice and attitudes in Sweden 1994. Acta Obstet Gynecol Scand 1996; 75: 932-940.

19. Piccinino LJ, Mosher WD. Trends in contraceptive use in the United States: 1982-1995. Fam Plann Perspect 1998; 30: 4-10.

20. Webb S, Holman DA. A survey of contraceptive use and unplanned pregnancy in Perth, Western Australia. Aust J Public Health 1992; 16: 382-386. 


\section{FE DE ERRATAS}

En el trabajo Conocimiento y uso de métodos anticonceptivos por la población femenina de una zona de salud. Maroto de Agustín A, Moreno Bueno M. ${ }^{\mathrm{a}} \mathrm{A}$, Rubio Moreno M. ${ }^{\mathrm{a}} \mathrm{M}$, Ortiz. Valle C y Escobar Rabadán F: 72 (6): 547-557, se produjeron erratas en la dirección de correo electrónico, en la tabla 2 y figuras 3 y 4 , las cuales deben ser como se publica a continuación.

Correo electrónico: fescobarr@medynet.com.
En la tabla 2 falta un signo «+» en la columna «quirúrgicos», el cual corresponde al «+» que indica la nota a pie de dicha tabla.

En la figura 3 se introdujo un error en la leyenda de los colores: dehe decir «No convive con pareja» $\mathrm{y}$ «Convive con pareja».

La figura 4 reflejaba la figura y leyenda de colores correspondiente a la figura 3 .

Figura 3

Conocimiento de métodos anticonceptivos en relación con la convivencia con pareja

$\%$

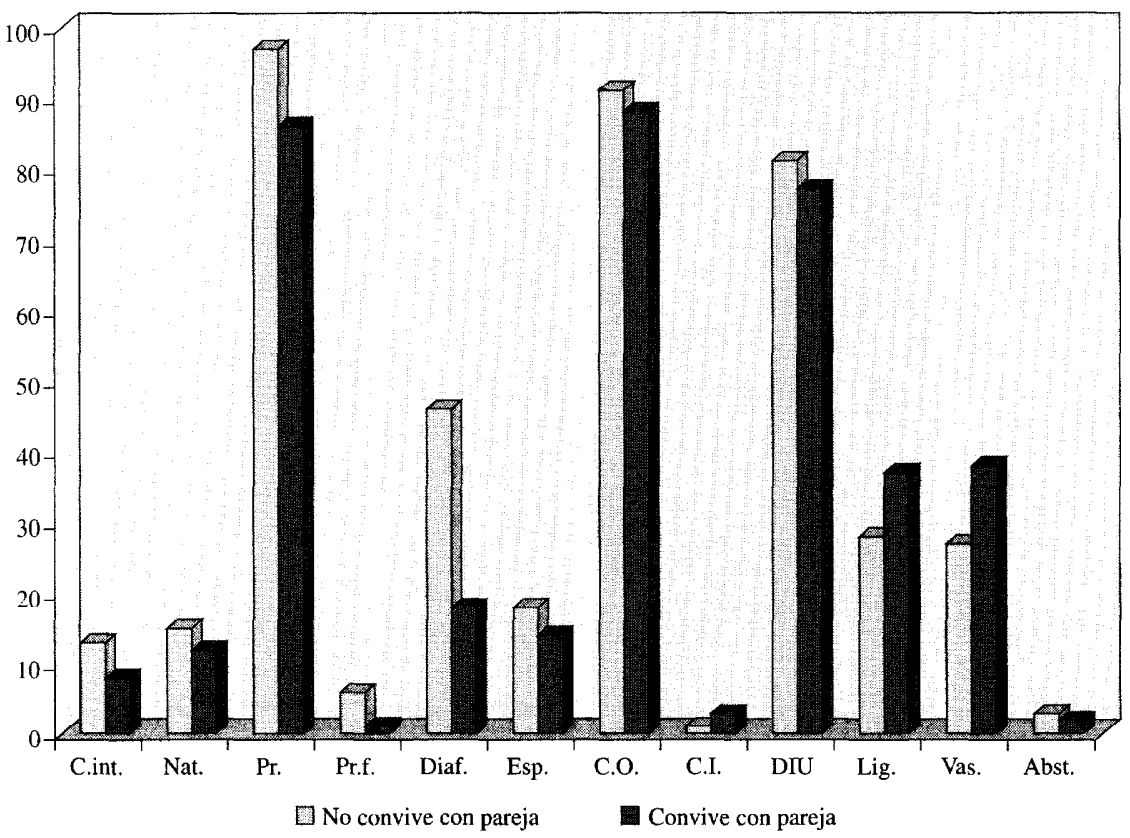

Nota: C.int.: coitus interruptus (NS)

Nat:: Métodos naturales (NS)

Pr.: Preservativo $\left(\mathrm{ji}^{2}=5,7 ; \mathrm{p}<0,05\right)$

Pr.f.: Preservativo femenino (NS)

Diaf.: Diafragma $\left(j i^{2}=15,1 ; \mathrm{p}<0,001\right)$

Esp.: Espermicidas (NS)
C.O.: Contraceptivos orales (NS)

C.I.: Contraceptivos inyectables (NS)

DIU: Dispositivo intrauterino (NS)

Lig.: Ligadura tubárica (NS)

Vas.: Vasectomía (NS)

Abst.: Abstinencia (NS) 
Figura 4

Conocimiento de métodos anticonceptivos en relación con el nivel de estudios

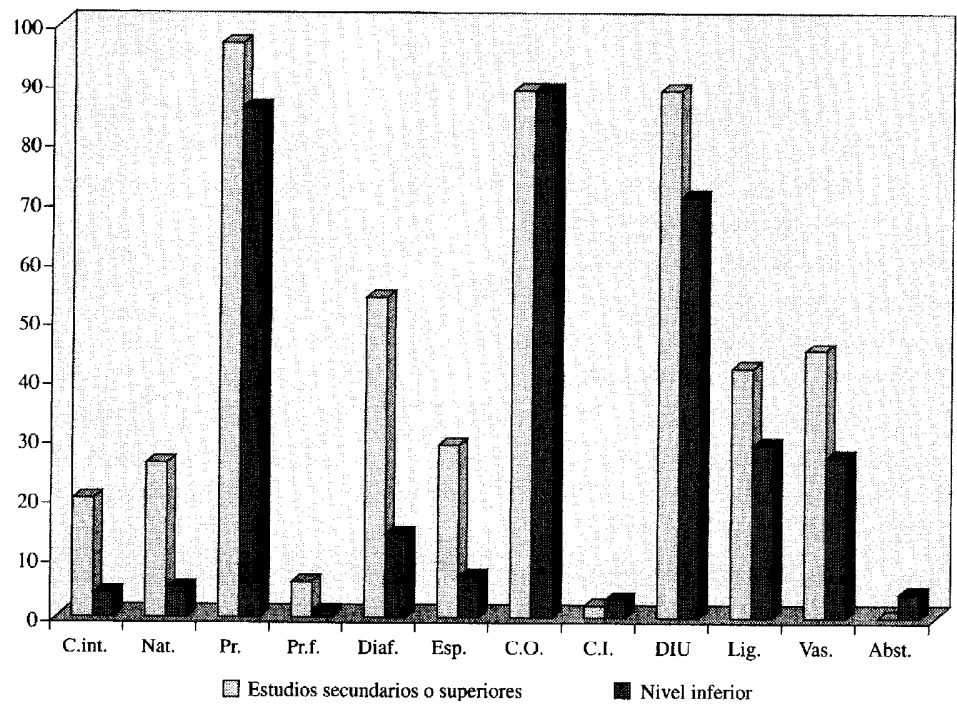

Nota: C.int.: coitus interruptus $\left(\mathrm{ji}^{2}=11,1 ; \mathrm{p}<0,001\right)$

C.O.: Contraceptivos orales (NS)

Nat.: Métodos naturales $\left(\mathrm{ji}^{2}=15,5 ; \mathrm{p}<0,001\right)$

Pr.: Preservativo $\left(\mathrm{ji}^{2}=5,3 ; \mathrm{p}<0,05\right)$

C.I.: Contraceptivos inyectables (NS)

Pr.f.: Preservativo femenino (NS)

Diaf.: Diafragma $\left(\mathrm{ji}^{2}=30.4: \mathrm{p}<0,001\right)$

Esp.: Espermicidas $\left(\mathrm{ji}^{2}=14,9 ; \mathrm{p}<0,001\right)$

Dispositivo intrauterino $\left(\mathrm{ji}^{2}=7,5, \mathrm{p}<0,01\right)$

Lig.: Ligadura tubárica (NS)

Vas.: Vasectomía $\left(\mathrm{ji}^{2}=7,4 ; \mathrm{p}<0,05\right)$

Abst.: Abstinencia (NS) 